\title{
A case report on the treatment of complex chronic pain and opioid dependence by a multidisciplinary transitional pain service using the ACT Matrix and buprenorphine/naloxone
}

This article was published in the following Dove Press journal: Journal of Pain Research

27 March 2017

Number of times this article has been viewed

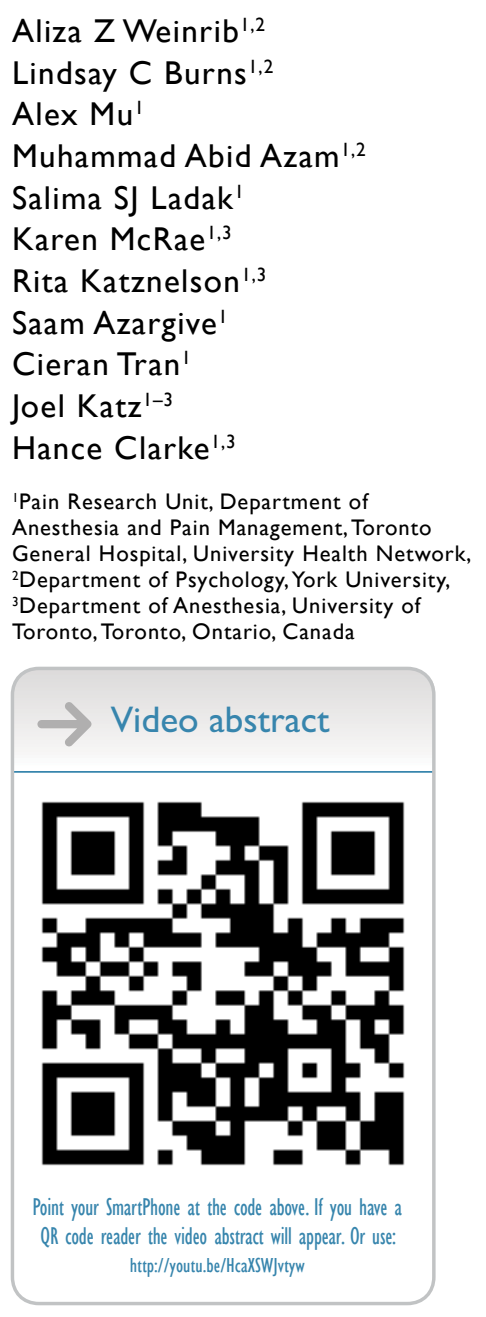

Correspondence: Aliza Weinrib and Hance Clarke Pain Research Unit, Department of Anesthesia and Pain Management, Eaton North 3-456, 200 Elizabeth Street, Toronto General Hospital, University Health Network, Toronto, ON M5G 2C4, Canada

Email aliza.weinrib@uhn.ca; hance.clarke@uhn.ca

\begin{abstract}
In an era of growing concern about opioid prescribing, the postsurgical period remains a critical window with the risk of significant opioid dose escalation, particularly in patients with a history of chronic pain and presurgical opioid use. The purpose of this case report is to describe the multidisciplinary care of a complex, postsurgical pain patient by an innovative transitional pain service (TPS). A 59-year-old male with complex chronic pain, as well as escalating long-term opioid use, presented with a bleeding duodenal ulcer requiring emergency surgery. After surgery, the TPS provided integrated pharmacological and behavioral treatment, including buprenorphine combined with naloxone and acceptance and commitment therapy (ACT) using the ACT Matrix. The result was dramatic pain reduction and improved functioning and quality of life after $40+$ years of chronic pain, thus changing the pain trajectory of a chronic, complex, opioid-dependent patient.
\end{abstract}

Keywords: transitional pain service, postsurgical pain, chronic pain, opioid dependence, opioid weaning, acceptance and commitment therapy

\section{Introduction}

Chronic pain is a highly prevalent and debilitating condition that results in suffering, disability, poor quality of life, and significant costs to the health care system. Patients with chronic pain are often prescribed long-term opioid therapy, despite its limited efficacy and growing concerns regarding the serious risks of opioid use (including side effects, dependence, and overdose mortality). ${ }^{1,2}$ Patients living with persistent pain and taking long-term opioids may require ever-increasing doses to maintain pain control due to opioid tolerance, while at the same time experiencing more and more side effects, including opioid-induced hyperalgesia, which paradoxically can increase pain sensitivity. ${ }^{2-4}$ The end result is an escalating cycle of pain and opioid use, which is of great concern to both patient and provider.

Primary care physicians and pain specialists are left with the significant challenge of providing pain relief to complex, chronic, opioid-dependent pain patients, while at the same time not feeding into the spiral of escalating opioid requirements. Moreover, when these patients need major surgery, they are at risk of a significant spike in opioid dose, due to the requirements of "acute-on-chronic" postsurgical pain management. ${ }^{5}$ According to data from Toronto General Hospital, ${ }^{6}$ patients on long-term opioid therapy for pain who undergo major surgery may leave hospital on up to three times their presurgical opioid dose, with little support or guidance for opioid weaning. To 
address the need for specialized, ongoing pain management for complex patients after surgery, we have created a novel transitional pain service (TPS), a hospital-based, specialist pain service with the mission of providing multidisciplinary pain management and support for opioid weaning for 3-6 months after major surgery. ${ }^{7}$

It has been recognized for some time that there is a need for comprehensive pain interventions (inclusive of pain medicine, psychology, and physiotherapy) that address the significant gap in care after surgery for patients at risk of chronic postsurgical pain. ${ }^{8}$ Accordingly, the TPS was developed at Canada's leading research hospital and one of its largest surgical centers, Toronto General Hospital, as a collaboration between clinicians and research scientists primarily from the disciplines of anesthesiology and pain psychology. ${ }^{7}$ The service was originally funded as a 1 year "demonstration project" by the Ontario Ministry of Health and Long Term Care (MOHLTC) to assess its feasibility, acceptability, and efficacy, and has since garnered stable funding as part of MOHLTC initiatives to improve pain management and opioid prescribing practices in Ontario, Canada.

One of the key goals of the TPS is to transform the critical period after surgery from a time when a new elevated opioid baseline is established (and likely thereafter maintained) to a time when postsurgical opioid weaning is supported and pain management is optimized using multimodal approaches, including behavioral strategies. To this end, patients are referred to the TPS if they have a history of presurgical chronic pain with or without opioid use, require high doses of opioid medication in hospital while reporting poor pain control, or have other risk factors for persistent postsurgical or complex pain and long-term opioid use. ${ }^{7}$ Pain specialists from multiple disciplines, including anesthesiology, nursing, psychology, and physiotherapy, work together in the TPS to create a multipronged plan addressing both pain and opioid weaning.

This report describes the postsurgical management of a patient with complex chronic pain and high-dose opioid dependence who came to the attention of the TPS team after urgent surgery. This case report describes how the TPS multidisciplinary team approached his care with the goal of managing his pain and opioid use.

\section{Participant and setting}

The collection and publication of TPS patient data were approved by the research ethics board at Toronto General Hospital. The patient gave written consent for the use of his data and publication of this case report.

\section{Initial surgical hospitalization}

Mr. P initially presented to the hospital's Emergency Department with severe hematemesis, which upon investigation revealed a bleeding duodenal ulcer. Notably, the likely cause of the ulcer was long-term intake of high-dose ibuprofen, a key indication that his efforts to self-manage his pain were running into difficulty, and that expert intervention was indicated.

Mr. P underwent a laparotomy to repair his ulcer, which successfully halted the bleeding; however, postoperative complications kept him in the hospital for several weeks. During this time, due to opioid tolerance, $\mathrm{Mr}$. $\mathrm{P}$ received high doses of opioid medication for postsurgical pain control. At the time of his referral to the TPS, he was receiving transdermal fentanyl ( $37.5 \mu \mathrm{g} /$ hour) and $65 \mathrm{mg}$ of oral hydromorphone per day, for a total daily morphine equivalent (MEQ) dose of $460 \mathrm{mg}$, which is more than double the current watchful dose of $200 \mathrm{mg}$ per day. ${ }^{9}$ Despite this dose of opioid medication, he continued to report severe "shooting, stabbing, and burning pain" in his back, legs, and joints, with a score of 4 on the ID pain questionnaire (greater than the clinical cutoff of 2), indicating his ongoing chronic pain had a neuropathic component. ${ }^{10}$ In addition, he reported mild postsurgical pain at this juncture.

\section{Presurgical pain history}

To understand how Mr. P came to be in this situation, it is important to review his pain history. In mid-adolescence, Mr. $\mathrm{P}$ developed acromegaly from a pituitary gland tumor leading to secretion of excess growth hormone, which resulted in abnormal growth of his hands, feet, and face. Although the tumor was removed in late adolescence, the acromegaly led to widespread osteoarthritis, kyphoscoliosis, and degenerative disc disease, which collectively caused him intermittent pain throughout his early adulthood, especially in his back and legs.

In 2004, Mr. P was diagnosed with fibromyalgia, a condition marked by widespread diffuse musculoskeletal pain, and in 2007, he was diagnosed with multiple sclerosis, resulting in peripheral neuropathy. Mr. P considers the fibromyalgia and peripheral neuropathy to be the primary sources of his pain over the past 30 years. With respect to his mental health, Mr. P has a diagnosis of bipolar disorder, which has been well controlled with mood stabilizing medication.

In addition to his pain history, Mr. $\mathrm{P}$ reported a history of substance use, one of the key risk factors for difficulties with opioid medication in people with chronic pain. He said that he had had a problem with alcohol "many years ago" and had stopped drinking on his own, as well as a period of benzodiazepine dependence 10 years ago that was followed by "mind boggling withdrawal". He had smoked 1.5-2.0 packs 
of cigarettes per day for 35 years and had successfully quit 10 years ago. He reported struggling to self-manage his pain with high doses of acetaminophen starting from the age of 19 years. In addition, Mr. P reported that he had been taking $3600-4800 \mathrm{mg}$ of ibuprofen daily for the past 5 years, likely contributing to the presenting surgical emergency.

Before his admission to hospital, he was taking 120 mg of oral oxycodone (180 mg MEQ) daily, just under the maximum safe dosage of $200 \mathrm{mg}$ MEQ per day according to contemporaneous opioid prescribing guidelines. ${ }^{9}$ Despite inadequate pain relief, $\mathrm{Mr}$. $\mathrm{P}$ reported that he had never resorted to purchasing drugs on the street. He stated:

It wasn't part of my framework [...] it is almost as if it didn't occur to me. I had a medical problem with pharmaceuticals.

I thought this was part of the medical world and needed to be solved with my doctor.

In that sense, his case is a notable presentation of opioid dependence in which all of the opioid medication he used was prescribed.

\section{Referral to the TPS}

Mr. P had a complex history of multiple pain conditions and polysubstance dependence, as well as a long history of high-dose opioid and nonsteroidal anti-inflammatory drug (NSAID) use, which came to a head with his presentation in hospital with a bleeding ulcer and subsequent surgery. After surgery, he suffered multiple complications, which prolonged his hospital stay for a total of 6 weeks. For example, he suffered a spontaneous pneumothorax in hospital followed by pigtail chest tube insertion, and subsequently a new diagnosis of chronic obstructive pulmonary disease. Once he was medically stable, his attending team was concerned about his high pain intensity and daily opioid use. A referral was made to the inpatient arm of the TPS for the management of his chronic and postsurgical pain, including optimization of his pain medication regimen, with the goal of reducing his opioid use. A TPS physician conducted an initial assessment at bedside at 5 weeks after surgery, and the advanced practice pain nursing team associated with TPS started visiting him daily to manage his pain medication, adding $600 \mathrm{mg}$ of gabapentin three times daily as a first step toward multimodal, opioid-sparing pharmacological pain management. Mr. P agreed to be followed by the TPS outpatient clinic once discharged from hospital (see Figure 1 for a timeline of the TPS interventions for this patient).

\section{Intervention and results Outpatient opioid-sparing pharmacological management}

Once Mr. P was out of the hospital, the first priority of pharmacological pain management was to wean him off of the fentanyl patch. Fentanyl - a drug associated with rapidly increasing tolerance and escalating doses - is a pain management option that should be reserved for cancer pain and palliative care; however, that being said, because of his

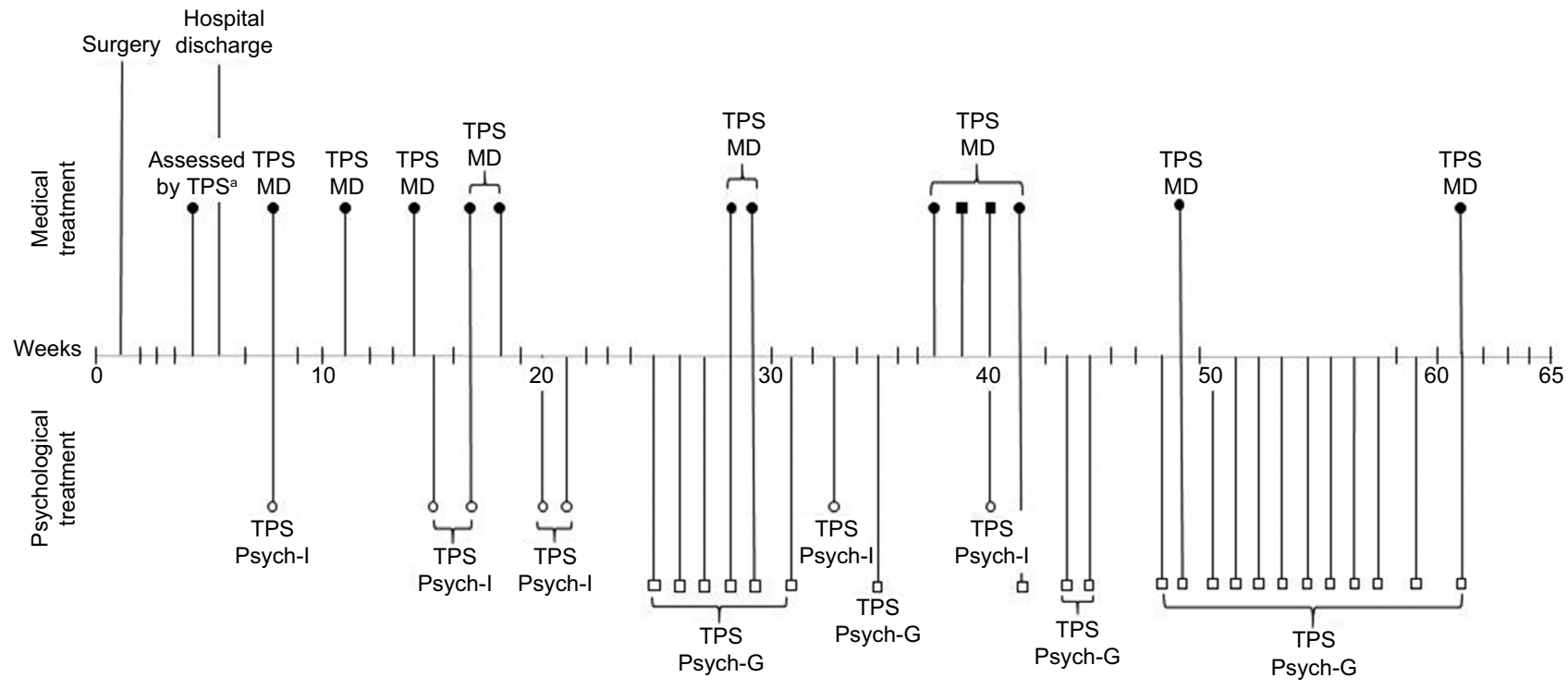

Figure I Timeline of patient's medical and psychological treatment by the TPS.

Notes: Closed circles indicate TPS MD appointment. Closed squares indicate buprenorphine/naloxone transition period. Open circles indicate clinical Psych-I appointment. Open squares indicate clinical Psych-G appointment. ${ }^{2}$ Assessment was conducted by TPS physician, and patient was seen by APS nurse daily until hospital discharge.

Abbreviations: APS, acute pain service; MD, medical doctor; Psych-I, psychologist individual (i.e., one-on-one appointment with psychologist); Psych-G, psychologist group (i.e., group session with psychologist and other pain patients); TPS, transitional pain service. 
postsurgical complications, Mr. P was NPO (i.e., unable to take food or medicine by mouth) for a time, which may have led to consideration of the fentanyl patch for pain control. Now that he was an outpatient, and moreover one with a history of substance dependence, fentanyl was an inappropriate long-term treatment. With steady effort and physician guidance, Mr. P was able to completely stop using the fentanyl patch and wean down from his in-hospital high of $460 \mathrm{mg}$ MEQ daily to under $300 \mathrm{mg}$ MEQ daily. This hard-won reduction in opioid use took 22 weeks after hospital discharge, and Mr. P was still well above the current maximum safe opioid dose of $200 \mathrm{mg}$ MEQ daily.

Mr. $\mathrm{P}$ described his postsurgical pain at the site of the incision as resolved completely by 13 weeks after surgery; however, he reported ongoing diffuse muscular pain due to fibromyalgia and peripheral neuropathy associated with multiple sclerosis that he rated as 7/10 in intensity on average. The TPS physicians continued to consider opioid-sparing adjunct medications to support him in weaning. The main classes of adjunct medication to be considered for the average patient include anticonvulsants, antidepressants (tricyclics and serotonin-norepinephrine reuptake inhibitors), NSAIDs, and cannabinoids. Mr. P continued to take the anticonvulsant gabapentin (600 mg three times daily); he could not take antidepressants because of a history of bipolar disorder, for which they are not indicated; he could not take NSAIDs due to his history of gastrointestinal (GI) bleed; he tried a cannabinoid, Nabilone (1 mg twice daily), with little effect. In addition, with the discontinuation of the fentanyl patch, his daily dose of hydromorphone $4 \mathrm{mg}$ (immediate release), which had begun at a maximum of 10 tablets per day (200 mg MEQ), had climbed to 15 tablets (300 mg MEQ) during the day as he was using more and then requesting early prescription refills, in addition to $9 \mathrm{mg}$ of oral hydromorphone controlled-release (45 MEQ) taken before bed so that he could sleep through the night without being woken by pain. At 8 months after surgery, he remained at $345 \mathrm{mg}$ MEQ daily with a pain score of 7/10 and, despite his best efforts, Mr. P was finding it very difficult to wean further.

\section{Physiotherapy}

Physiotherapy is an important component of the management of chronic pain, as well as postsurgical recovery. Accordingly, the TPS team offered Mr. P the opportunity to receive treatment from the TPS physiotherapist as part of his recovery program, which many patients find highly beneficial. However, Mr. P declined as he was already engaged in physiotherapy through a community-based organization with a weekly group exercise program for patients with multiple sclerosis.

\section{Behavioral intervention using acceptance and commitment therapy (ACT)}

Mr. P expressed an interest in behavioral treatment: "I wanted change and a deeper understanding of myself [...] a place to honestly talk about my struggles with medication and get help". Mr. P first met with the TPS clinical psychologist 2 weeks after discharge from hospital. He participated in five individual counseling sessions in the first 4 months after hospital discharge; at that time, he joined a weekly behavioral pain management group and opted to attend group sessions in lieu of individual therapy, for a total of 22 group sessions attended in the 61 weeks since surgery. The group became an important place for Mr. P:

The benefit of the group is that it introduces another strategy for coping with pain, bridged my isolation and provided a sense of comfort. Meeting and talking with people with similar experiences helped me to learn how to handle difficulty better and more productively and to rethink my own relationship to pain meds.

The individual and group behavioral treatments in the TPS are grounded in ACT, a contemporary form of behavior therapy that is a part of the recent wave of therapies that incorporate mindfulness and acceptance-based strategies into treatment ${ }^{11}$ for a wide variety of behavioral problems, including the treatment of depression and anxiety, ${ }^{12}$ psychosis, ${ }^{13}$ and substance misuse (including opiate addiction). ${ }^{14}$ ACT has gained strong research support for the treatment of chronic pain, according to the American Psychological Association, Division $12 .{ }^{15}$ When it comes to pain management, ACT emphasizes mindfully observing pain sensations in the body at each moment, while noticing the thoughts and feelings that are stirred up by pain, and "making space" for these pain-related sensations, thoughts and feelings to be a part of one's inner experience. In ACT interventions, participants are taught through a series of experiential exercises and teaching metaphors that when pain cannot be eliminated by medical means, "opening up" to pain sensations can paradoxically lead to relief. Patients can practice "opening up" (i.e., acceptance of pain sensations) during mindfulness meditation. Mindfulness is an ancient practice for calming the mind ${ }^{16}$ that has garnered much evidence of effectiveness in the treatment of pain, ${ }^{17}$ and is fully integrated into ACT. In addition to the mindfulness component of ACT, which helps people to live with pain with less suffering, there is 
also a strong behavioral emphasis on taking action based on personal values (e.g., being a loving partner or being active and self-reliant). The ACT approach can be summarized in this statement: "I am here now, accepting the way I feel, noticing my thoughts, while doing what I care about". ${ }^{18}$ For people living with pain, this means observing pain and one's reaction to pain, while engaging in daily meaningful activities that are connected to deeply held personal values. ACT has been shown repeatedly to lead to improved functioning for people living with medical illness and pain, ${ }^{19,20}$ with a positive association between increased mindfulness and improved functioning. ${ }^{21}$

\section{The ACT matrix}

A visual tool, known as "the Matrix", has been developed for teaching the ACT model. ${ }^{22,23}$ Using the matrix, core ACT principles can be taught quickly and effectively, making it particularly suitable for brief interventions. The matrix is fundamentally based on two axes, a vertical axis and a horizontal axis (Figure 2). The vertical axis makes the distinction between: 1) the inner world of pain, emotions, thoughts, and urges, and 2) the outer world that we can see, hear, taste, smell, and touch, and in which we can take action. The horizontal axis distinguishes between the actions that are motivated by avoidance, such as pain avoidance (away moves) and actions that are part of living a rich, engaged, and meaningful life (toward moves). The clinician asks four key questions (Figure 2). The matrix intervention begins by highlighting the ineffectiveness and the personal cost of predominately avoidance-based behavioral patterns at baseline and supports the individual to build new patterns of engaged and purposeful behaviors driven by personal values and priorities.

With the help of the pain psychologist and the use of an ACT Matrix diagram, Mr. P learned to recognize when he was "stuck" (as symbolized by the arrows on the left of Figure 3 ) in a vicious cycle of pain and pain avoidance - and his "away moves" included taking opioid medication in order to avoid the suffering that comes with opiate withdrawal. At that point, escaping from pain and withdrawal symptoms had become the focus of his life: "Being out of pain can become a goal in itself. Pursuing that goal can take the whole day". From a behavioral perspective, this reflects the narrowing of the behavioral repertoire that results when behavior is driven by pain avoidance, with a clear cost to approach behavior (i.e., engagement in personally meaningful life activities) - and yet, unfortunately, without the elimination of pain. ${ }^{24}$ He noticed that he had been using opioids to treat not only his pain but also the emotional suffering that both contributed to and exacerbated his pain. When he was engaged in pain-avoidance behavior, Mr. P described himself as retreating to his apartment, sitting on the same spot on the couch, alone, taking excessive opioid medication, watching $\mathrm{TV}$, and sleeping. In contrast, he developed clarity as to what his value-based approach behavior would look like: being productive with respect to his work as an artist, going out to visit family and friends, and participating in his weekly physiotherapy group for individuals living with multiple sclerosis - a meaningful "toward move" for him, even though it sometimes led to more pain in the short term. Week by week, in group therapy in particular, he became clear on his

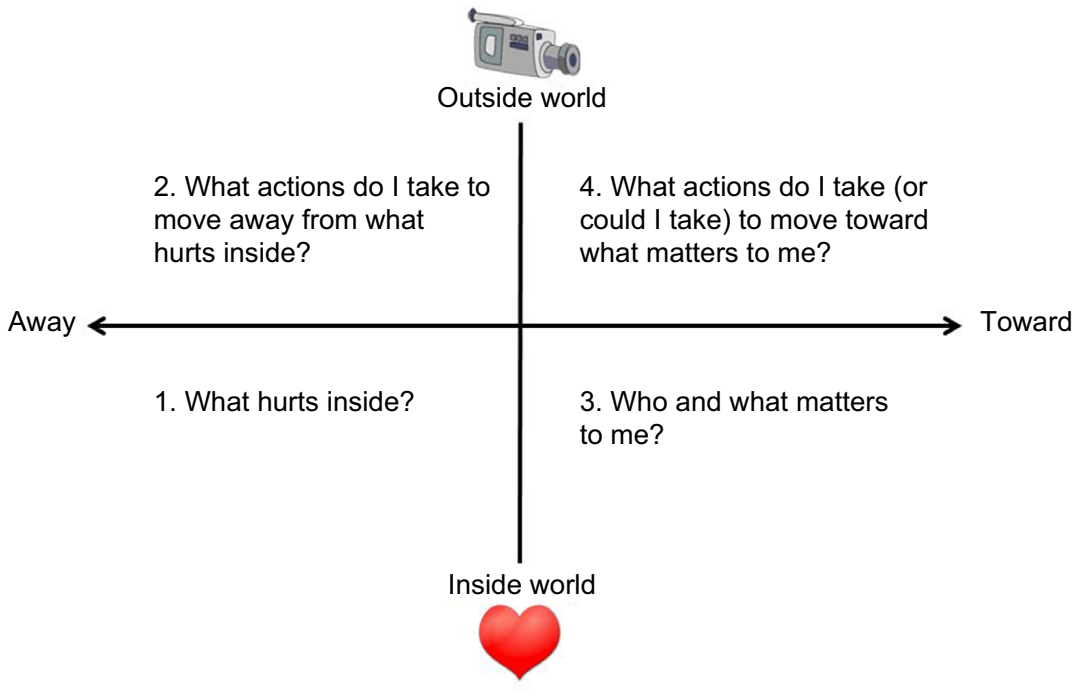

Figure 2 The ACT matrix,,$^{22,23}$ a visual tool for teaching the ACT approach, is applied here to pain management. Abbreviation: ACT, acceptance and commitment therapy. 


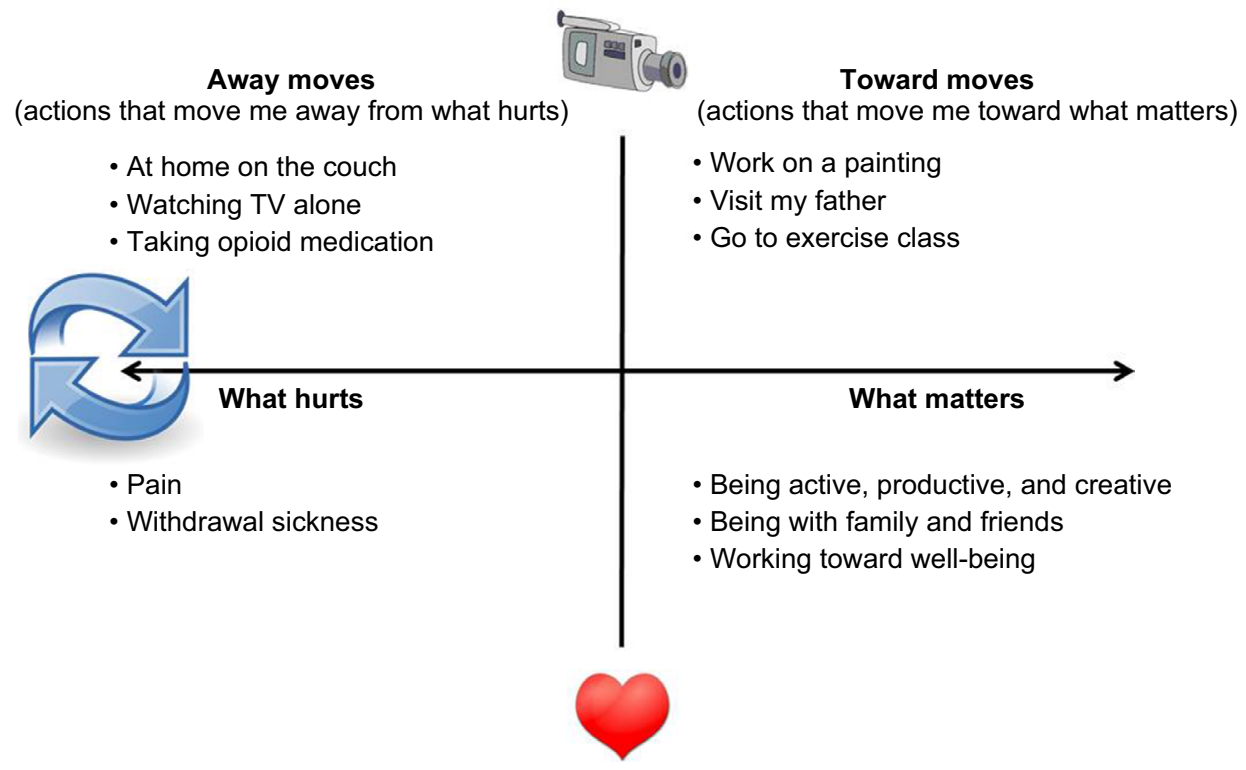

Figure 3 Example of the ACT matrix used by Mr. P.

Notes: The heart represents the "inside world" - the realm of private, internal experience including pain (what hurts) and personal values (what matters). The video camera represents the "outside world" of observable actions. The psychologist asks the key question, "What would you see yourself do on camera (to move toward X or away from Y)?" to focus awareness on behavior. The left side of the diagram represents pain-based avoidance behavior (i.e., moving away from what hurts), whereas the right side of the diagram represents values-based approach behavior (i.e., moving toward what matters).

Abbreviation: ACT, acceptance and commitment therapy.

personal priorities, identified steps that he wanted to take that were aligned with these priorities, shared successes in taking action based on what mattered to him, and processed set-backs in a supportive context.

\section{Mindfulness practice}

In addition to the ACT matrix, each group meeting included time for mindfulness practice. Mr. P practiced observing the sensations of pain here and now just as they are, letting go of the psychological struggle with the pain, and intentionally expanding awareness beyond the boundaries of the pain to include the breath, the world around us, and the chosen activity of the moment. Mr. P began practicing mindfulness formally and informally daily, in addition to practicing with the group, and found it helpful in coping with the pain. He was initially skeptical about whether he could focus on anything other than the pain, which he called "compelling". However, at the end of treatment, he stated, "I think many people with pain might not be open to trying it, but when I tried it, I learned that mindfulness is an effective tool for me". Mr. P reported that mindfulness practice gave him a new perspective on his pain, created a sense of calm, and with practice he felt more able to "engage with my life".

\section{A new pharmacological approach}

Mr. P had made progress in the 8 months after surgery. He had weaned his opioid use from $460 \mathrm{mg}$ MEQ daily down to
345 mg MEQ daily, a significant drop but still considerably over the current "watchful" maximum safe dose of $200 \mathrm{mg}$ MEQ and his baseline presurgical dose of $180 \mathrm{mg}$ MEQ. ${ }^{9} \mathrm{He}$ had participated earnestly in the behavioral pain treatment program run by the TPS, and joined a weekly physiotherapy group for patients with multiple sclerosis. Nevertheless, as he articulated, he was struggling to further wean his opioid medication:

Pain medication became a brutal rollercoaster of opiates and their constant companion - withdrawal sickness. And yet incredibly, the pain that I lived with constantly was never truly soothed despite the very large quantities of pain pills that I was taking. The pain medicine was actually causing more pain, and the initial physical pain itself was never truly addressed. This was the dark side of the opiates. The addiction was now serving only itself.

It became clearer over this time that Mr. P was using opioids to alleviate/avoid his opioid withdrawal symptoms, perhaps more than he was using it to relieve his pain. This was more than a pain problem - it was problematic opioid drug dependence. Many patients who have been on long-term opioids rely on their opioid regimen not only to reduce pain but more so to avoid the physiologic withdrawal that may ensue if the opioid medications were to be stopped or a dose missed. It is important to note that not all of these patients are good candidates for a rotation to buprenorphine and naloxone. 
Mr. P was an excellent candidate because his psychological distress regarding his medication use was significant. Clinically we made no promise of improved pain control at the outset, simply the potential to improve his quality of life by decreasing his psychological distress.

The TPS physicians suggested a trial of a sublingual formulation of buprenorphine/naloxone to Mr. P as the next step in his recovery. Buprenorphine is a partial $\mu$ opioid receptor agonist with a high receptor affinity compared with other opiates and a long half-life, which has made it an effective drug to treat opioid dependence. Unlike full $\mu$ opioid receptor agonists, such as methadone, buprenorphine acts like an antagonist at higher doses by occupying receptors but only partially activating them, resulting in a ceiling effect that prevents major side effects, such as respiratory depression, leading to a decrease in the risk of overdose. Naloxone, an opioid receptor antagonist, is included to deter crushing and injecting of the pill in cases of drug diversion.

Mr. P was fearful of transitioning to buprenorphine/ naloxone partly due to going into withdrawal. However, the TPS worked as a team to answer his questions, and provided information on the benefits of converting to a stable buprenorphine/naloxone regimen as well as counseling on what to expect with regard to pain and withdrawal during buprenorphine/naloxone induction and stabilization. On the first day, Mr. P was brought into the office in withdrawal (on the Clinical Opiate Withdrawal Scale, he scored 16), ${ }^{25}$ and started on an induction dose of $4 \mathrm{mg}$ of the buprenorphine/naloxone compound, assessed 2 hours later and given another dose of $4 \mathrm{mg}$, and then sent home with another $4 \mathrm{mg}$ to a maximum of $12 \mathrm{mg}$ on the first day of his induction. At the end of the second day, Mr. P was still struggling with withdrawal symptoms, so was prescribed $24 \mathrm{mg}$ of the buprenorphine/naloxone compound once daily with followup in 5-7 days. After 1 week, Mr. P pronounced that he "felt like Superman":

The pain in my legs is under control for the first time in 30 years. The major change in my life is unbelievable. For the first time in 40 years, I am not thinking about pain pills first thing in the morning. I am free of the hamster wheel.

Mr. P's transition to buprenorphine/naloxone was accompanied by an unanticipated rapid and dramatic reduction in his pain ratings and sustained improvement in levels of engagement in meaningful life activities (Figure 4).

Now that he was no longer suffering from daily withdrawal symptoms, and his pain was under control, Mr. P was able to fully reap the rewards of the behavioral skills he had learned in the pain management group. During one group session after his transition to buprenorphine/naloxone, Mr. P described a difficult episode of pain, and joked, "Rather than swimming in my pain, I went to the swimming pool". Rather

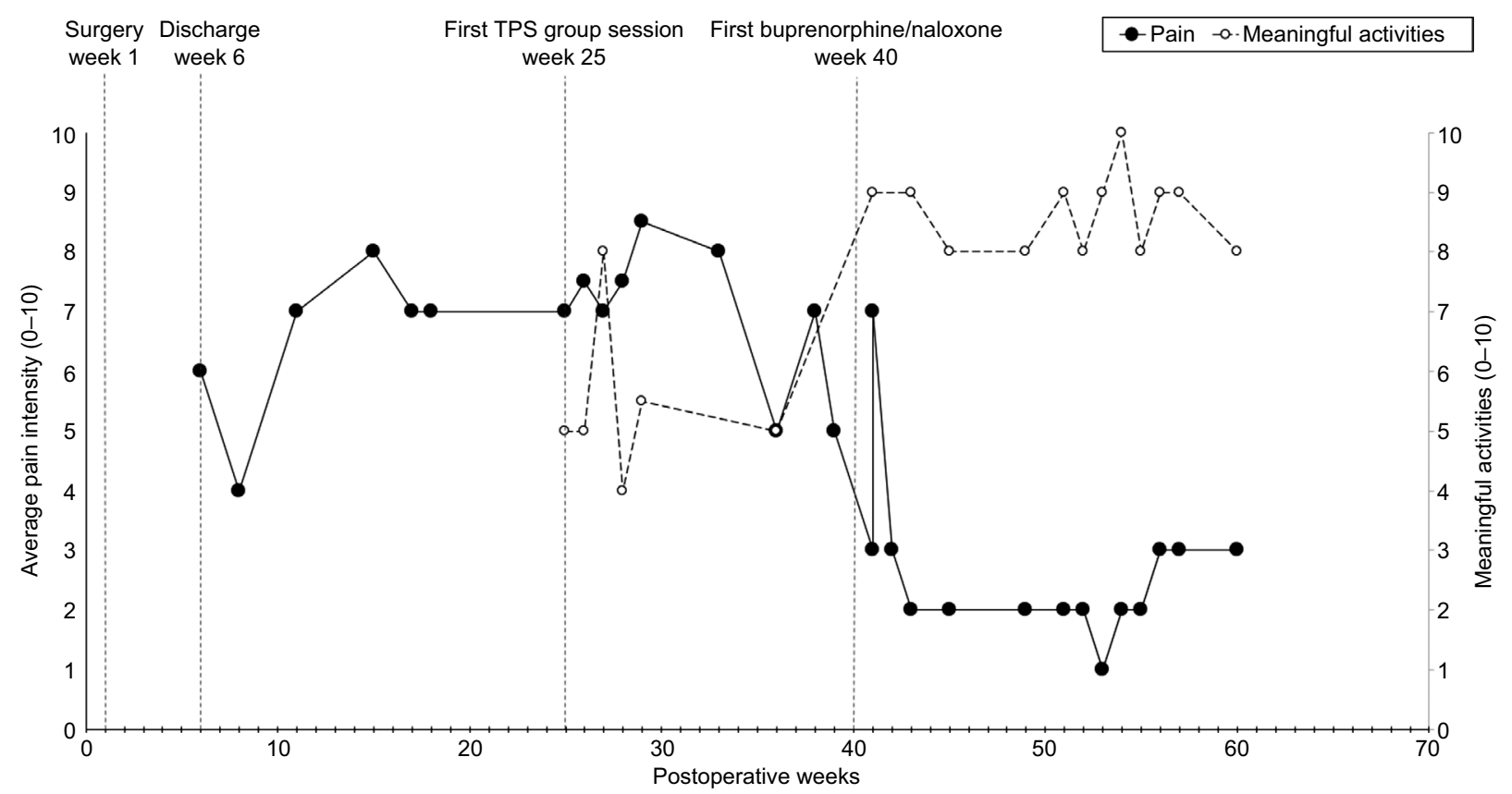

Figure 4 Average pain intensity scores and engagement in meaningful activities over follow-up period.

Notes: Pain was measured using a numeric pain rating scale ranging from 0 (no pain) to 10 (worst pain imaginable). Level of engagement in meaningful activities was assessed from the start of group therapy using a numeric rating scale ranging from 0 (not doing anything that matters) to 10 (doing everything that matters).

Abbreviation: TPS, transitional pain service. 
than sitting alone in his apartment, overwhelmed by pain, self-medicating, and in retreat, he got himself moving, connected with his neighbors, and fully appreciated the present moment. This is an example of how mindfulness and behavior change can work hand-in-hand, leading to new possibilities in terms of functioning and quality of life. Through a transition to buprenorphine/naloxone, coupled with the development of self-management strategies, Mr. P was able to overcome his opioid dependence, reduce his pain to manageable levels, and restore his engagement with life.

That's what has been given back to me: the opportunity to be engaged in my life, to participate with my friends and family, to pursue my career, to simply be connecting with a life that is a good life.

\section{Discussion}

This report presents the case of a patient with complex chronic pain on a path of escalating opioid use paired with inadequate pain relief. The TPS multidisciplinary pain management team (i.e., physicians, psychologists, nurses, and physiotherapists) provided Mr. P with personalized, expert, and ongoing consultation in the months after surgery to reduce pain, provide alternatives to escalating use of shortacting opioids, and give him a behavioral "toolkit" that supported functioning and quality of life.

The postoperative hospital setting provides an important catchment system for patients struggling with, or at high risk of developing, chronic pain conditions and opioid dependence, and offers the opportunity for timely and coordinated multidisciplinary intervention. Mr. P was identified after surgery as struggling with intense pain and opioid dependence and with the help of the TPS, his pain trajectory was radically altered. It is difficult to imagine a case of this complexity being handled successfully in primary care, but all too often that is the default since 1 in 5 individuals suffer from chronic pain, ${ }^{26}$ despite one specialized pain clinic for every 51,600 people with chronic pain in Canada. ${ }^{27}$ Improved pain and addiction management resources within the hospital setting are critical to addressing patient needs and can have a significant impact in terms of public health moving forward.

For patients with chronic pain, misuse of opioid medication is common with a recent review reporting that $\sim 25 \%$ of pain patients misuse their opioids. ${ }^{28}$ Patients taking longterm opioids are susceptible to developing tolerance, which results in: 1) strong urges to take more opioids, 2) the need for increasing dosages of opioids to gain the same amount of pain relief, and 3) withdrawal symptoms (including muscle aches, abdominal cramping, and irritability), all of which can lead to overuse of opioids to alleviate these symptoms in a self-perpetuating cycle. ${ }^{1,2}$ Moreover, a frequently overlooked adverse effect of prolonged opioid use is increased pain sensitivity. Long-term exposure to opioids can actually make individuals more sensitive to pain through neuroplastic changes in the peripheral and central nervous systems, a phenomenon known as opioid-induced hyperalgesia, which may have been at play in this case, given the sharp drop in pain reported after the transition to buprenorphine/naloxone. ${ }^{29}$ Patients suffering from pain as well as opioid tolerance and withdrawal need our empathy and support in order to break the self-perpetuating cycle of opioid use, and find a new path forward with good quality of life, even if pain persists.

Closing the gap in poorly managed pain and excessive opioid prescribing will require systemic changes to our methods of delivering treatment to individuals with chronic and complex pain. Current gaps in care include 1) difficulties in identifying patients in timely way (i.e., ideally before the pain becomes chronic), 2) insufficient physician training in opioid prescribing, 3) insufficient physician training in multimodal pain management, 4) inadequate access to consultations with pain specialists, 5) lack of access to multidisciplinary teams including dedicated pain clinics, and 6) lack of funding for such teams, especially for psychological services that are fundamental to this approach. Mr. P's case highlights that a buprenorphine/naloxone combination can be a helpful medication for appropriate candidates in a chronic pain program, where proper screening, diagnosis, and open discussions with patient and family are involved to make the best clinical decision regarding treatment options. Even more importantly, his successful case demonstrates the value of treatment programs such as the Toronto General Hospital's TPS in closing the gap in pain management and opioid dependence by leveraging the organizational framework and relevant patient catchment of a hospital setting, and intervening across the spectrum of complex acute to chronic pain conditions with an integrated, multimodal approach.

\section{Acknowledgments}

Funding for the Transitional Pain Service provided by the Ontario Ministry of Health and Long-Term Care. JK is supported by a Canadian Institutes of Health Research Canada Research Chair in Health Psychology at York University. HC is supported by a Merit Award from the Department of Anesthesia, University of Toronto.

\section{Disclosure}

The authors report no conflicts of interest in this work. 


\section{References}

1. Chou R, Turner JA, Devine EB, et al. The effectiveness and risks of long-term opioid therapy for chronic pain: a systematic review for a national institutes of health pathways to prevention workshop. Ann Intern Med. 2015;162(4):276-286.

2. Savage SR. Long-term opioid therapy: assessment of consequences and risks. J Pain Symptom Manage. 1996;11(5):274-286.

3. Fletcher D, Martinez V. Opioid-induced hyperalgesia in patients after surgery: a systematic review and a meta-analysis. Br J Anaesth. 2014; 112(6):991-1004.

4. Bannister K, Dickenson AH. Opioid hyperalgesia. Curr Opin Support Palliat Care. 2010;4(1):1-5.

5. Wenzel JT, Schwenk ES, Baratta JL, Viscusi ER. Managing opioidtolerant patients in the perioperative surgical home. Anesthesiol Clin. 2016;34(2):287-301.

6. Clarke, H. Transitional Pain Medicine: novel pharmacological treatments for the management of moderate to severe postsurgical pain. Expert Review of Clinical Pharmacology. 2016;9(3):345-349.

7. Katz J, Weinrib A, Fashler SR, et al. The Toronto General Hospital Transitional Pain Service: development and implementation of a multidisciplinary program to prevent chronic postsurgical pain. J Pain Res. 2015;8:695-702

8. Katz J, Seltzer Z. Transition from acute to chronic postsurgical pain: risk factors and protective factors. Expert Rev Neurother. 2009;9(5):723-744.

9. Canadian guideline for safe and effective use of opioids for chronic noncancer pain. Canada: National opioid use guideline group (NOUGG); 2010 [cited 2017 January 25]. Available from: http://nationalpaincentre. mcmaster.ca/opioid. Accessed Feburuary 23, 2017.

10. Portenoy R. Development and testing of a neuropathic pain screening questionnaire: ID Pain. Curr Med Res Opin. 2006;22(8):1555-1565.

11. Hayes SC, Villatte M, Levin M, Hildebrandt M. Open, aware, and active: contextual approaches as an emerging trend in the behavioral and cognitive therapies. Annu Rev Clin Psychol. 2011;7:141-168.

12. Forman EM, Herbert JD, Moitra E, Yeomans PD, Geller PA. A randomized controlled effectiveness trial of acceptance and commitment therapy and cognitive therapy for anxiety and depression. Behav Modif. 2007;31(6):772-799.

13. Bach P, Hayes SC. The use of acceptance and commitment therapy to prevent the rehospitalization of psychotic patients: a randomized controlled trial. J Consult Clin Psychol. 2002;70(5):1129-1139.

14. Hayes SC, Wilson KG, Gifford E V, Byrd M, Gregg J. A preliminary trial of twelve-step facilitation and acceptance and commitment therapy with opiate addicts. Behav Ther. 2004;35:667-688.
15. American Psychological Association. Acceptance and Commitment Therapy EST Status for Chronic or Persistent Pain: strong research support. Society of Clinical Psychology (Division 12) [Internet]. American Psychological Association; 2013 [cited Jun, 1 2015]. Available from: http://www.div12.org/psychological-treatments/disorders/chronic-orpersistent-pain/acceptance-and-commitment-therapy-for-chronic-pain/. Accessed January 23, 2017.

16. Young S. Natural Pain Relief: How to Soothe and Dissolve Physical Pain with Mindfulness. Sounds True; 2011.

17. Zeidan F, Grant JA, Brown CA, McHaffie JG, Coghill RC. Mindfulness meditation-related pain relief: evidence for unique brain mechanisms in the regulation of pain. Neurosci Lett. 2012;520(2):165-173.

18. Moran DJ. Building Safety Commitment. Joilet, IL: Valued Living Books; 2013.

19. McCracken LM, Morley S. The psychological flexibility model: a basis for integration and progress in psychological approaches to chronic pain management. J Pain. 2014;15(3):221-234.

20. Dindo L, Recober A, Marchman JN, Turvey C, O’Hara MW. Oneday behavioral treatment for patients with comorbid depression and migraine: a pilot study. Behav Res Ther. 2012;50(9):537-543.

21. McCracken LM, Gauntlett-Gilbert J, Vowles KE. The role of mindfulness in a contextual cognitive-behavioral analysis of chronic pain-related suffering and disability. Pain. 2007;131(1):63-69.

22. Polk K, Schoendorff B, Wilson KG. The ACT Matrix. Oakland, CA: New Harbinger Publications, Inc.; 2014.

23. Polk K, Schoendorff B, Webster M, Olaz F. The Essential Guide to the ACT Matrix: A Step-by-step Approach to using the ACT Matrix Model in Clinical Practice. Oakland, CA: New Harbinger Publications, Inc.; 2016.

24. Wicksell RK, Olsson GL. Predicting and preventing chronic postsurgical pain and disability. Anesthiosology. 2010;113(6):1260-1261.

25. Wesson D, Angeles L. The clinical opiate withdrawal scale (COWS). 2016;(April).

26. Schopflocher D, Taenzer P, Jovey R. The prevalence of chronic pain in Canada. Pain Res Manag. 2011;16(6):445-450.

27. Fashler SR, Cooper LK, Oosenbrug ED, et al. Systematic review of multidisciplinary chronic pain treatment facilities. Pain Res Manag. 2016; 2016:1-19.

28. Vowles KE, McEntee ML, Julnes PS, Frohe T, Ney JP, van der Goes DN. Rates of opioid misuse, abuse, and addiction in chronic pain: a systematic review and data synthesis. Pain. 2015; 156(4):569-576.

29. Lee M, Silverman S, Hansen H, Patel V, Manchikanti L. A comprehensive review of opioid-induced hyperalgesia. Pain Physician. 2011;(14): 145-161.
Journal of Pain Research

\section{Publish your work in this journal}

The Journal of Pain Research is an international, peer reviewed, open access, online journal that welcomes laboratory and clinical findings in the fields of pain research and the prevention and management of pain. Original research, reviews, symposium reports, hypothesis formation and commentaries are all considered for publication.

\section{Dovepress}

The manuscript management system is completely online and includes a very quick and fair peer-review system, which is all easy to use. Visit http://www.dovepress.com/testimonials.php to read real quotes from published authors. 